\title{
Multiplex and On-site PCR detection of swine diseases based on the microfluidic chip system
}

\section{CURRENT STATUS: UNDER REVIEW}

BMC Veterinary Research $\triangle$ BMC Series

Bin Zhou

Nanjing Agricultural University

zhoubin@njau.edu.cnCorresponding Author

ORCiD: https://orcid.org/0000-0001-7279-9489

Yan Jiang

Nanjing Customes

Yue Wu

Nanjing Agricultural University

Shan Jiang

Nanjing,Customs

Kaiming Wang

Nanjing,Customs

Dexin Zeng

Nanjing,Customs

Gan Chen

Jiangganshan Agricultural Science and Technology Park Management Committee

DOI:

$10.21203 / \mathrm{rs} .2 .21078 / \mathrm{v} 1$

\section{SUBJECT AREAS}

Small Animal Medicine

\section{KEYWORDS}

swine disease, microfluidic chips, PCR, multiplex and on-site detection 
Abstract

Background $\square$ At present, the process of inspection and quarantine starts with sampling at the customs port, continues with transporting the samples to the central laboratory for inspection experiments, and ends with the inspected results being fed back to the port. This process takes a rather long time, has the risks of degradation of biological samples [32] and generation of pathogenic microorganisms [33], and does not meet the rapid on-site detection demand [34]. Therefore, development of a technology for rapid and high- throughput detection of pathogenic microorganisms at the customs port is of great significance. This study was to develop a microfluidic chip to be applied to rapid high-throughput swine disease detection with higher accuracy and lower risk of spreading pathogenic microorganisms during transportation.

Results: PCR technology has the advantages of high accurate and sensitivity in disease detection, clinical testing and food quarantine, so it plays an important role in customs inspection. However, the traditional PCR detection instrument has a large size, is time-consuming and has strict requirements on the experimental environment, which greatly limit its application in on-site testing. In this paper, the clinical samples of four swine diseases were detected by a portable and rapid microfluidic PCR system, which could achieve a on- site real-time quantitative PCR detection. Eight clinical samples were detected together on the microfluidic chip in the system, and the detection results were obtained in about an hour. The detection limit of this microfluidic PCR detection system was as low as 1 copies/ $\mu \mathrm{L}$. The results show that the high sensitivity and specificity of the microfluidic PCR detection system in disease detection will play an important role in customs inspection and quarantine during customs clearance.

Conclusion: The microfluidic PCR detection system established in this study could meet the requirements for rapid detection of samples at the customs port

The new method can avoid the risky process of transporting the samples from the sampling site to the testing lab, and drastically reducing the inspection cycle, and would enable parallel inspections on one chip which greatly raising the efficiency of inspection.

Full Text 
Due to technical limitations, full-text HTML conversion of this manuscript could not be completed.

However, the manuscript can be downloaded and accessed as a PDF.

Tables

Due to technical limitations, tables are only available as a download in the supplemental files section Figures

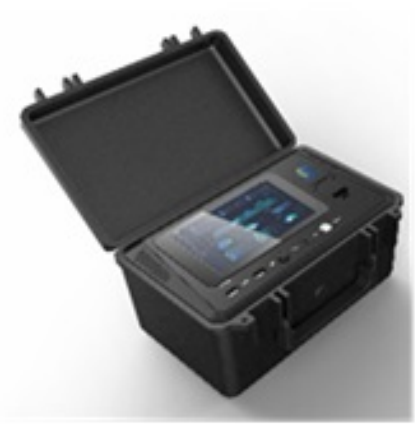

(A)

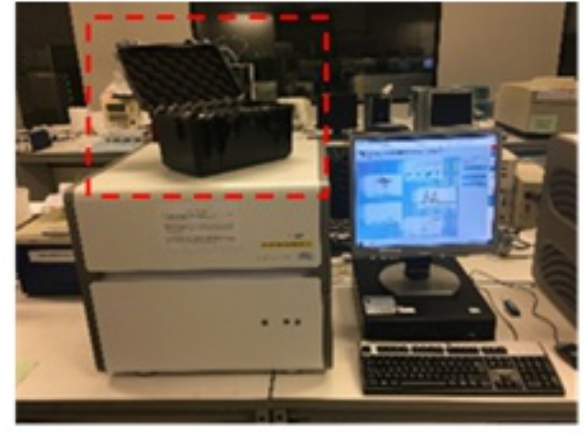

(B)

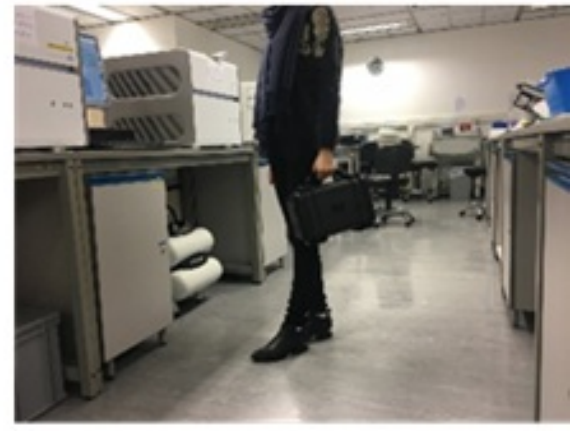

(C)

Figure 1

(a) The portable rapid microfluidic PCR system; (b) Size comparison of the microfluidic PCR instrument (within the red dotted box) with the traditional large PCR instrument (Roche 480); (c) Demonstration of the portable design of the instrument. 

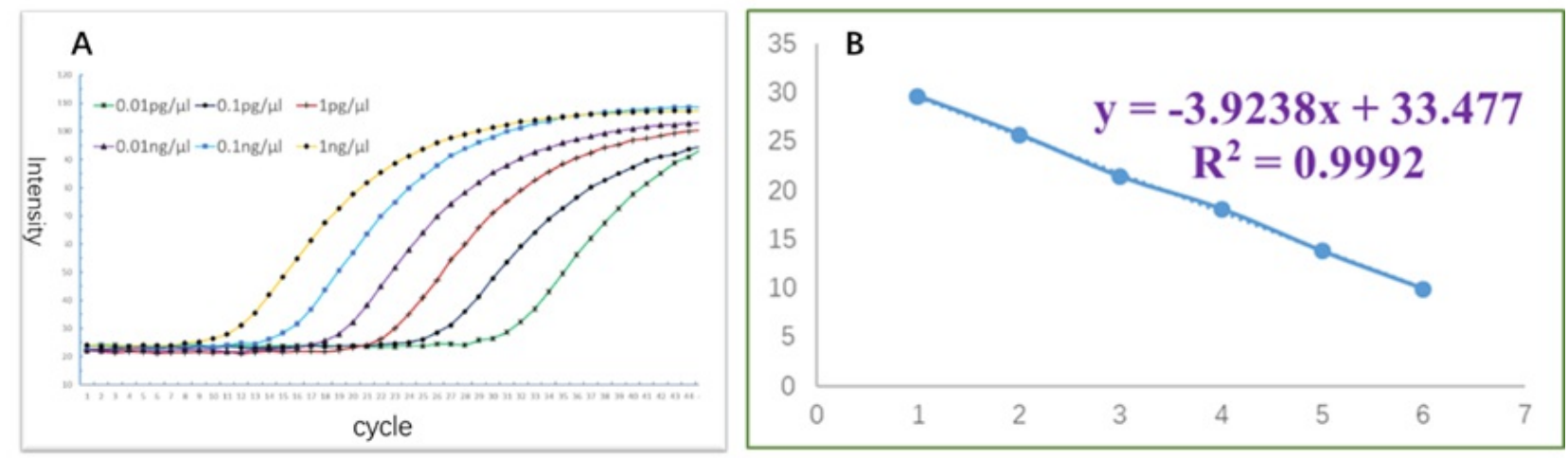

Figure 2

(a) The sensitivity test of the portable rapid microfluidic PCR system; (b) The linear correlation curve of the dynamic range.

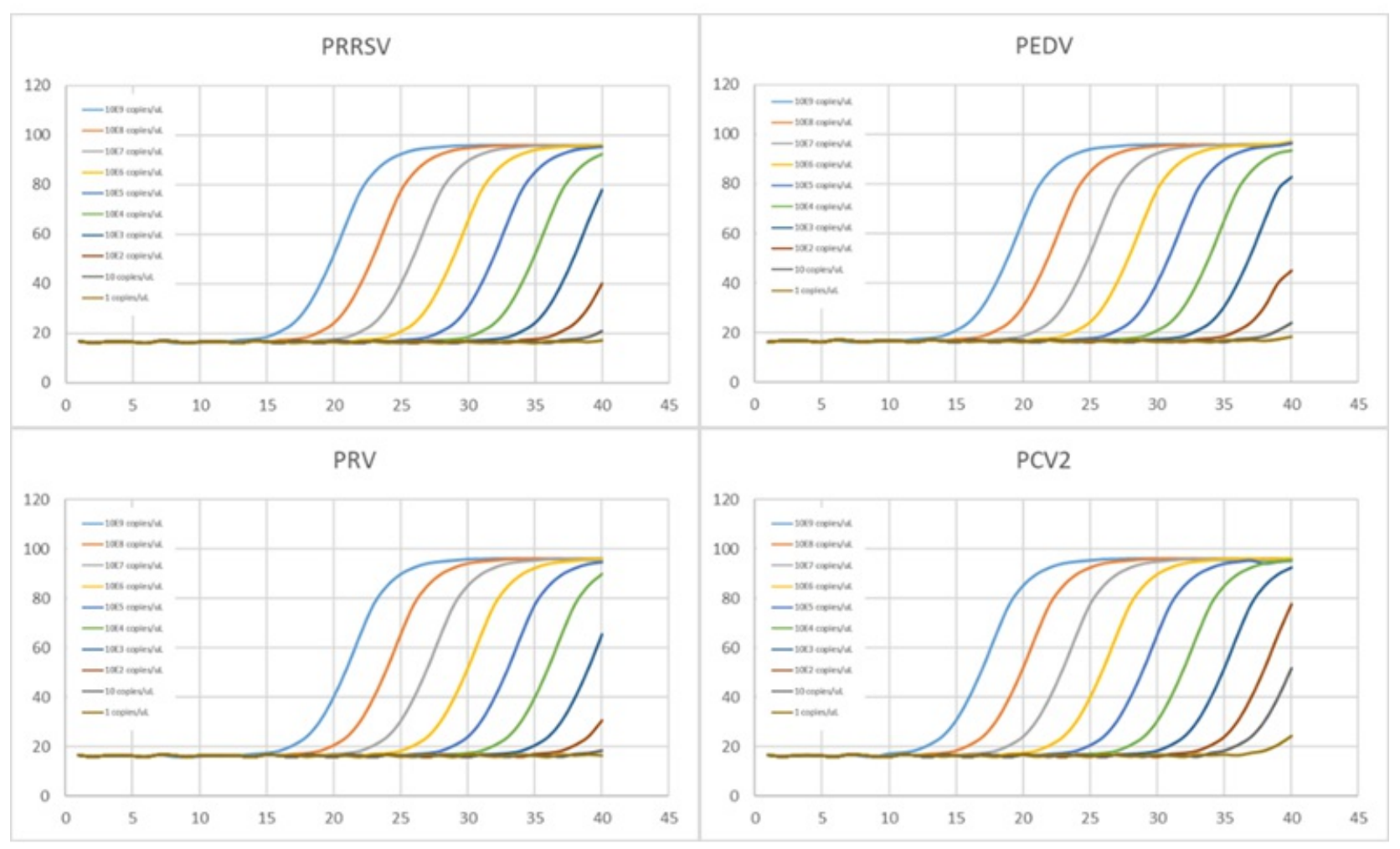

Figure 3

Sensitivity test of the PCR protocols of the four swine disease viruses. 

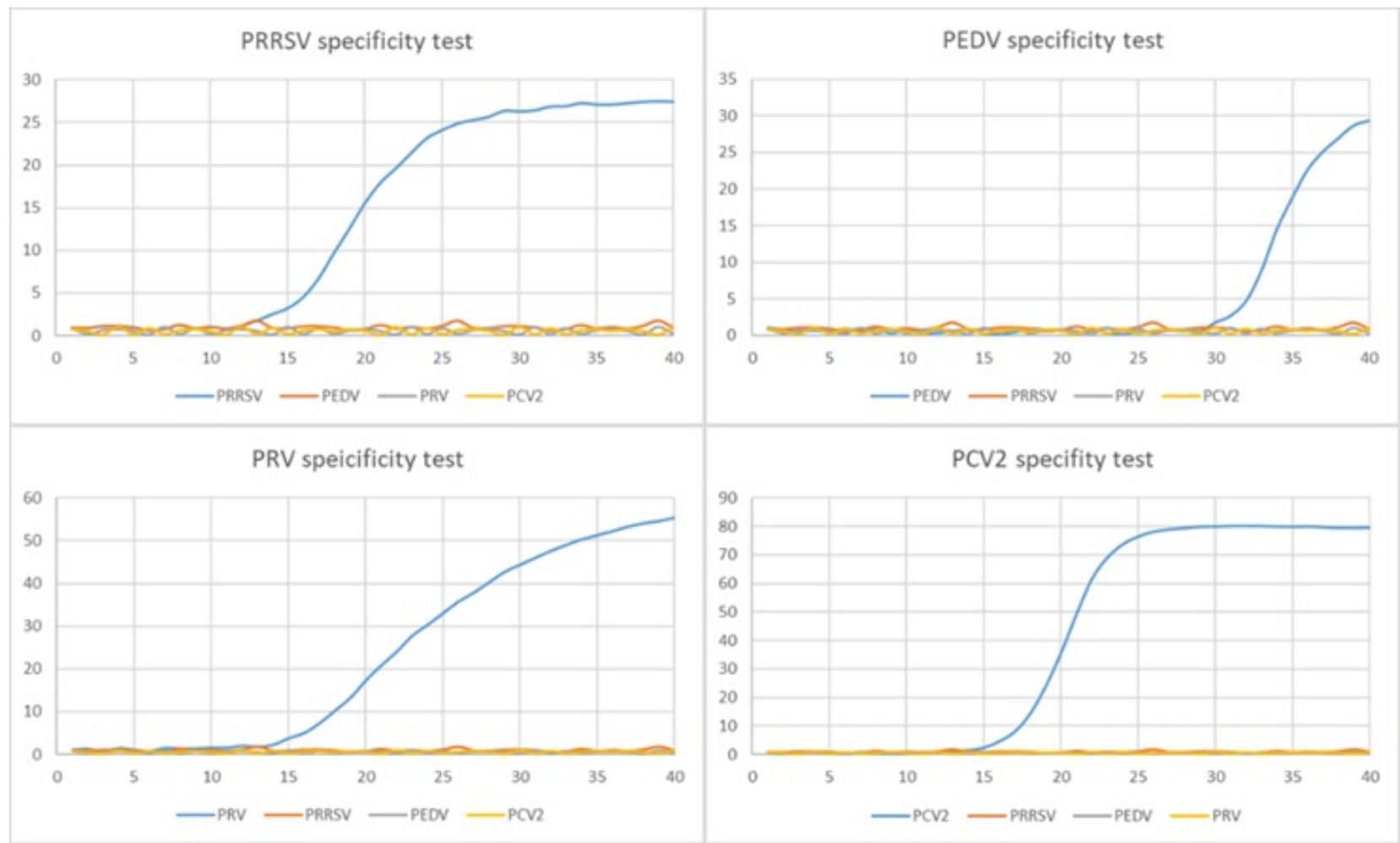

Figure 4

Specificity test of the four swine disease viruses on the chip. 


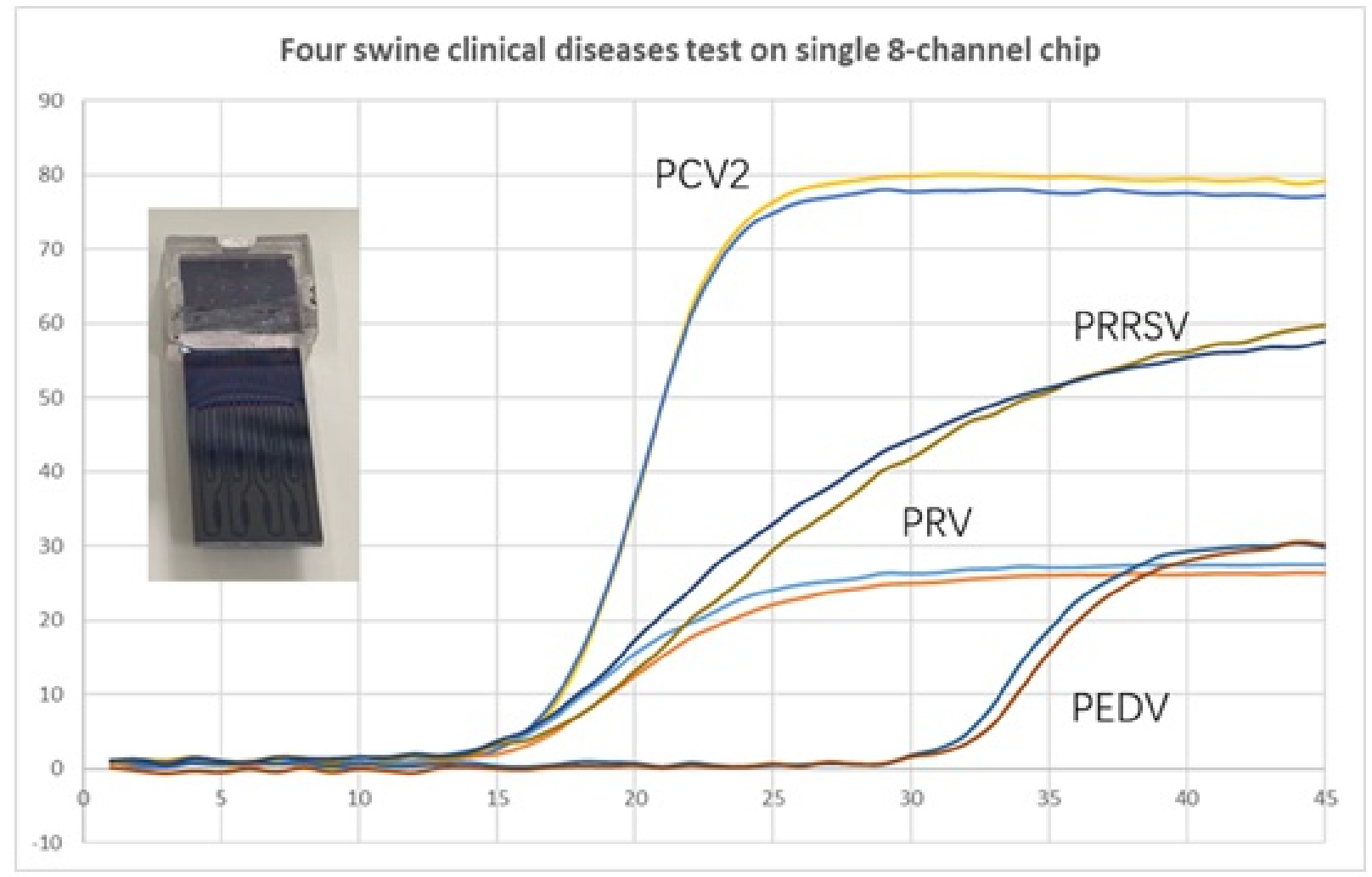

Figure 5

Parallel detection results of four clinical samples of swine diseases on the 8-chamber microfluidic chip.

\section{Supplementary Files}

This is a list of supplementary files associated with this preprint. Click to download.

Table1.xlsx 